\title{
Réflexion sur la place l'action collective dans la revendication d'une infrastructure routière : le cas de la route 175
}

\author{
Jean-Guillaume Simard ${ }^{1}$ \\ Université du Québec à Chicoutimi
}

\section{Introduction}

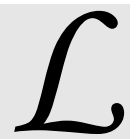
e 22 août 2002, les gouvernements fédéral et provincial tournaient une page de l'histoire du Saguenay-Lac-Saint-Jean en annonçant la réalisation du doublement de la route 175 dans la réserve faunique des Laurentides. Après plus de trente ans de revendications, le projet faisait enfin l'unanimité et ce, autant de la part des deux paliers gouvernementaux que du milieu régional. L'annonce de ce projet, qui fut réellement qualifié de victoire de la région, a tôt fait d'effacer le passé trouble et difficile qui a entouré les revendications. Pendant trois décennies, le projet de doublement de la route 175 a fait partie du paysage régional, constamment martelé par les groupes de pression et largement diffusé par l'action des médias.

Cet article se veut une réflexion sur la place de l'action collective dans la revendication d'une infrastructure routière. Au cours des dernières décennies, la mobilisation entourant l'implantation de grands équipements a souvent fait place à différentes formes de contestation : celles rattachées à la diminution de la qualité de vie qui découle de cette implantation (généralement associées au NIMBY ${ }^{2}$ ), mais également à des enjeux plus généraux comme le bien commun et l'environnement ${ }^{3}$.

Le projet de doublement de la route 175 faisant le lien entre la région du Saguenay-Lac-Saint-Jean et Québec s'intègre dans ce courant, mais dans une logique inversée. Ainsi, la mobilisation entourant le projet s'est exprimée sous forme de revendications où les pouvoirs publics en contestaient le bien-fondé. Cette vision positive de l'infrastructure, rattachée principalement aux notions de sécurité et de développement économique, a perduré dans le paysage régional pendant plus de trente ans. L'aspect très localisé de l'entreprise est un facteur expliquant le succès mitigé de la revendication dans le temps.

Nous tenterons de circonscrire le contexte dans lequel s'est insérée la mobilisation afin d'évaluer la place de l'action collective dans l'acceptation du projet.

Pour établir la chronologie des événements, nous avons assis cette recherche sur une abondante revue de presse retraçant les faits marquants du dossier dans la période comprise entre 1972 et 2004. De plus, différentes entrevues furent réalisées afin de confirmer la succession des événements, mais également afin préciser les nuances dans l'argumentaire des acteurs.

\section{Mise en contexte du projet}

C’est en 1944 que fut amorcé l'aménagement d'un lien routier entre la région du Saguenay-Lac-SaintJean et Québec dans la réserve faunique des Laurentides. Avec l'essor économique et démographique qu'avait connu la région au cours des années 1930 et 1940, notamment en raison des différentes avancées dans le secteur de l'aluminium, une route efficace et sécuritaire s’imposait alors aux autorités comme étant nécessaire au développement de la région. Avant cette date, le transport s'effectuait principalement par train et par bateau.

Il revint à monsieur Antonio Talbot, alors ministre de la Voirie pour le gouvernement Duplessis et député du comté de Chicoutimi, d'approuver la construction de ce lien routier. Le montant alloué pour le projet dépassait les deux millions et demi de dollars et représentait 
la plus importante somme jamais accordée par le ministère de la Voirie. Le chantier constituait un effort d'ingénierie incroyable pour l'époque. Environ 600 personnes travaillèrent de l'hiver 1944 à l'été 1948 pour la construction de la route. Un million de mètres cubes de terre furent grugés et l'on procéda à la construction de 200 ponts et ponceaux afin de frayer un chemin dans la réserve faunique des Laurentides. La route allait être inaugurée quatre ans après le début des travaux, soit le 8 juillet 1948.

Au cours des décennies qui suivirent la construction de la route, de nombreuses critiques furent formulées de la part d'intervenants de la région. Compte tenu du terrain accidenté qui caractérise le tracé de la route, de nombreux accidents vinrent ternir la réputation de l'axe routier. Plusieurs facteurs, outre l'aspect topologique, en étaient responsables : conditions atmosphériques extrêmes, entretien difficile lors des périodes hivernales, présence d'orignaux aux abords de la route, vitesse excessive, etc.

\section{Naissance du projet de doublement de la route 175 (1972-1987)}

C’est en 1972, porté par la Société nationale des Québécois, que le projet de route à quatre voies divisées dans la réserve faunique des Laurentides commença à s'articuler et à gagner en popularité dans la région du Saguenay-Lac-Saint-Jean. Le Conseil régional de développement (CRD) ne s'opposa pas à cette initiative, malgré des divergences d'opinions en ce qui concerne le développement du réseau routier régional. Toutefois, le ministère des Transports se montra plutôt froid en regard à la construction d'une route à quatre voies divisées. Le Ministère exigea, pour la construction de ce type de route, un débit de circulation variant entre 10000 et 12000 véhicules par jour. La situation en 1972 était loin de satisfaire ces exigences : environ 2200 véhicules étaient recensés en moyenne quotidiennement. Même pour la période estivale, le débit quotidien arrivait à peine à franchir les 5500 véhicules.

L'aspect de la sécurité prit beaucoup de place dans l'argumentaire des défenseurs du projet. Par contre, on renchérit rapidement avec le développement économique que pourrait induire cette infrastructure. Rappelons que la croissance économique qu'a connue le Saguenay-Lac-Saint-Jean après la Seconde Guerre mondiale ne permettait pas de diversifier l'économie régionale où la majorité des activités de production se retrouvait en amont des filières de production ${ }^{4}$. Fortement dépendante de la demande internationale, l'industrie primaire de la région subit de nombreuses pertes d'emplois reliées au courant de modernisation des installations et de rationalisation du personnel qui s'ensuivit au cours des années 1980. Parallèlement à ce déclin de l'emploi dans les secteurs traditionnels, le secteur tertiaire profita d'une progression soutenue. Par contre, l'abondante création d'emplois dans ce secteur n'arriva pas à compenser la perte des emplois bien rémunérés caractéristiques du secteur primaire. Voilà le contexte dans lequel mûrit le projet.

\section{Le Ministère exigea, pour la construction de ce type de route, un débit de circulation variant entre 10000 et 12000 véhicules par jour.}

\section{L’apogée du militantisme (1988-1993)}

Au début des années 1980, il n’était presque plus question du projet de route à quatre voies divisées dans la réserve faunique des Laurentides. Les investissements consentis par le gouvernement du Québec pour la réfection de la route de même que l'argumentaire limité des militants en furent les principales causes. Le projet de doublement de la route 175 renaquit en 1988 sous l'initiative des conseils municipaux des villes de Jonquière et Chicoutimi (avec l'aide d'une station radiophonique locale). Il en résulta le groupe Accès-Bleuets, un groupe de pression qui milita pendant plus de 15 ans pour le projet.

Rapidement diffusée à travers les différents médias d'information de la région, l'initiative populaire ne fit pas l'unanimité. En effet, la partie jeannoise de la région ne partageait pas les mêmes objectifs quant au développement du réseau routier régional. Ainsi, l’opération Accès-Bleuets souleva peu d'intérêt au LacSaint-Jean où la population, à l'image de ses représentants politiques, voulait s'en tenir aux priorités déjà établies (dont la route 175 ne faisait pas partie). À ce titre, nous pouvons mentionner le projet d'autoroute Alma-La Baie et celui de la route 169 ceinturant le Lac-Saint-Jean.

À la suite des moyens de pression controversés entrepris au cours de la période estivale de $1989^{5}$, le groupe Accès-Bleuets se vit dans l'obligation de se 
retirer de la scène publique. Ces événements poussèrent le groupe à revoir sa stratégie, d'une part, en remaniant l'exécutif de l'organisme et, d'autre part, en développant davantage son argumentaire qui jusquelà était principalement associé à l'aspect sécurité.

Toutefois, les pressions sociales engendrées par la mobilisation portèrent fruit. En mai 1989, le ministère des Transports annonçait la réalisation d'une étude d'opportunité d'un lien routier entre les régions de Québec et du Saguenay-Lac-Saint-Jean ${ }^{6}$. Cette même étude favorisa l'amélioration ponctuelle de la route, reconnaissant toutefois la dangerosité de celle-ci (tableau 1).

\section{Tableau 1 - Nombre d'accident survenu sur la route 175 (du $\mathrm{km} 60$ au $\mathrm{km} \mathrm{217)}$ entre 1985 et 2001*}

\begin{tabular}{|lccc|}
\hline Type d'accident & $\begin{array}{c}\text { Nombre } \\
\text { d'accidents } \\
\text { 1985-1989 }\end{array}$ & $\begin{array}{c}\text { Nombre } \\
\text { d'accidents } \\
\mathbf{1 9 9 3 - 1 9 9 7}\end{array}$ & $\begin{array}{c}\text { Nombre } \\
\text { d'accidents } \\
\mathbf{1 9 9 7 - 2 0 0 1}\end{array}$ \\
\hline Mortels & 47 & 24 & 22 \\
Blessés graves & 91 & 76 & 50 \\
$\begin{array}{l}\text { Blessés légers } \\
\begin{array}{l}\text { Dommages } \\
\text { matériels }\end{array}\end{array}$ & 358 & 290 & 228 \\
seulement & 1153 & 1094 & 744 \\
\hline Total & $\mathbf{1 6 4 9}$ & $\mathbf{1 4 8 4}$ & $\mathbf{1 ~ 0 4 4}$ \\
\hline
\end{tabular}

Source : Ministère des Transports.

* Les données pour la période comprise entre 1990 et 1992 n’ont pu être rassemblées.

\section{La reprise politique du dossier (1993-...)}

L'année 1993 marque un tournant important dans le dossier. En effet, la reconnaissance officielle de la route 175, par la Commission royale des transports, comme faisant partie du réseau routier national ouvrait la porte à un partenariat fédéral-provincial pour le financement de sa réfection. Une enveloppe de 25 millions de dollars fut d'ailleurs octroyée par le gouvernement fédéral pour la réfection de la route, celleci étant conditionnelle à un investissement similaire de la part du gouvernement du Québec. Cet épisode marqua le début d'une confrontation entre les deux paliers de gouvernement pour courtiser l'électorat régional.
Le 8 avril 1998, contre toute attente, le premier ministre du Québec d’alors, Lucien Bouchard, annonça la réalisation d'une seconde étude d'opportunité ${ }^{7}$ afin d'évaluer la pertinence, les échéances et les modes de réalisation du projet de doublement de la route 175 . L'orientation empruntée par le gouvernement pour cette étude reposait sur l'évaluation des besoins de la route en termes de volume de déplacement et de sécurité des usagers ainsi que sur les retombées économiques induites. Cette étude prônait la réalisation d'une route à quatre voies divisées dans la réserve faunique des Laurentides.

Cela mena le gouvernement du Québec à mettre sur pied un plan stratégique d'intervention de 143 millions \$ investis sur une période de 10 ans (20002010). Selon l'échéancier, le projet de route à quatre voies divisées serait complété en 2020. Fait intéressant à noter, le projet serait réalisé uniquement avec des fonds québécois.

Le militantisme du député conservateur du comté de Chicoutimi-Le Fjord de l'époque, André Harvey, n'est pas étranger au nouveau sujet d'actualité que représentait le financement de l'infrastructure. À la fin de l'année 2000, le gouvernement fédéral (représenté par le Parti libéral) reconnut le dossier de la route 175 comme étant prioritaire. C'était le prix à payer pour s'adjoindre le député vedette de Chicoutimi. À ce titre, la mise sur pied du Fonds canadien sur l'infrastructure stratégique fut perçue par les intervenants de la région comme un engagement formel du gouvernement fédéral à la suite de cette reconnaissance accordée au projet.

Cette démarche politique aboutit à l'annonce, par les premiers ministres fédéral (Jean Chrétien) et provincial (Bernard Landry), de la réalisation du projet de doublement de la route 175 dans la réserve faunique des Laurentides à l'aube des élections provinciales en 2002. Le projet était alors prévu sur un échéancier de cinq ans et les coûts de réalisation assumés à parts égales entre Québec et Ottawa à travers le Fonds canadien sur l’infrastructure stratégique.

Selon les termes du contrat, le gouvernement du Québec serait le maître d'œuvre du projet. Celui-ci fera d'ailleurs l'objet d'une seconde annonce " officielle » le 7 mai 2004 avec, cette fois-ci, les premiers ministres Paul Martin et Jean Charest ${ }^{8}$. 


\section{Route à quatre voies divisées ou développement régional ?}

Deux éléments sont à souligner dans le déroulement de ce dossier. D'une part, le symbolisme entourant le projet et, d'autre part, l'objet de la revendication luimême.

\section{Le discours émotif entourant le projet n'a donné que plus d'impact à la revendication.}

\section{Le symbolisme du projet}

Le projet de doublement de la route 175 a toujours été perçu de manière très positive dans la région du Saguenay-Lac-Saint-Jean. Cette vision du projet lui a permis de perdurer en se rapprochant constamment des préoccupations de la population régionale. Tout d'abord, sa mise en service fut associée très tôt à la résolution du problème que représentait la sécurité défaillante sur l'axe routier. Ensuite, d'autres considérations rattachées aux manques ressentis au niveau de l'économie de la région se sont ajoutées graduellement, faisant de la route un catalyseur inestimable pour le Saguenay-Lac-Saint-Jean. Sans nier l'importance que revêt un bon système de transport sur l'économie d'une région, aucune contestation ne semble avoir été menée envers la nature même du projet. Ainsi, on acceptait d'emblée l'apport que pouvait avoir la réfection de l’axe routier.

Il est vrai qu'il existait des divergences d'opinions concernant le projet de la route 175 . Cependant, celles-ci se regroupaient autour des priorités de développement du réseau routier régional. Sans s’afficher en défaveur du projet, les opposants favorisaient d'autres projets routiers dans la région ${ }^{9}$. Personne n'en questionnait le bien-fondé.

Rappelons que les principales raisons autour desquelles s'organisait la contestation d'infrastructures routières se concentraient sur la qualité de vie diminuée par la présence de celle-ci (pollution, bruit, visibilité réduite, etc.). La mobilisation qui entoura le prolongement de la route 73 dans le secteur de Stoneham résume bien cet état des choses ${ }^{10}$. La construction de cette route, sensée être la première étape du doublement de la route 175, fit l'objet d'une contestation soutenue de la part des riverains, retardant considérablement sa réalisation. Mentionnons que ce tronçon était le lieu de nombreux accidents mortels.

La réserve faunique des Laurentides représente un territoire de $6800 \mathrm{~km}^{2}$ de superficie séparant la région du Saguenay-Lac-Saint-Jean et Québec. Cette zone, qui possède une vocation récréotouristique, recense très peu d'établissements résidentiels permanents. Aucun mouvement de protestation ne s'est formé envers le projet et ce, au cours des trente années qu'a duré la revendication. Seul les aspects positifs ont alors été exposés à la population régionale. Ainsi, il était accepté que la réfection de la route 175 aurait comme conséquence la diminution du nombre d'accidents, l'abolition de la barrière que représente la réserve faunique, l'augmentation du nombre d'industries dans la région, l’accroissement du tourisme, etc.

Même si ces conséquences, souhaitables pour la région il va sans dire, n'étaient pas automatiquement assurées par le doublement de la route, la conscientisation et la diffusion qui en ont été faites reflétaient une certaine vision déterministe de l'infrastructure. Ainsi, l'association constante effectuée entre la réfection de la route et la résolution de problèmes conjoncturels fut assimilée par la population régionale comme un reflet réaliste de la situation.

Ces sujets très émotifs que sont la sécurité, l'équité et le développement économique ont soulevé les sensibilités de la population régionale. Bien que leur résolution relève d'une multitude de facteurs, il semble qu'une certaine complaisance se soit manifestée autour du projet au point tel qu'il fut identifié comme une partie (peut-être trop) importante de la solution. Et ceci, autant par la population locale que par les autorités gouvernementales. Il s'agissait d'une solution somme toute facile face à des problèmes beaucoup plus profonds et complexes.

À ce titre, le discours émotif entourant le projet n'a donné que plus d'impact à la revendication, celle-ci possédant une justification morale. Ainsi, le projet fut développé sur la base de l'amélioration des conditions de sécurité routière. Avec le constat que des accidents mortels survenaient sur l'axe et que l'aménagement d'une route à quatre voies divisées pouvait faire diminuer le nombre d'accidents, le projet atteignit une position d’invulnérabilité. 
S’ajoutait à cela un idéal d'équité faisant du lien routier la dernière barrière à franchir pour que la région du Saguenay-Lac-Saint-Jean se considère comme égale à ses voisines nord-américaines dans un contexte, de plus en plus présent, de mondialisation des marchés. Le doublement de la route 175 représentait l'outil indispensable lui permettant de faire face au nouveau millénaire.

\section{L'objet de la revendication}

Ce que nous constatons du déroulement de ce projet, c'est l'ambiguïté de l'objet de la revendication. Contrairement à d'autres expériences de mobilisation ayant une vision plus globale du développement, le cas de la route 175 se veut plus localisé en portant son objet sur une infrastructure. Les possibilités offertes par la route en constituent l'argumentaire.

L'amélioration de la sécurité défaillante de la route 175 , bien qu'elle ait constitué l'amorce du projet, a vu son importance diminuer au détriment d'enjeux plus économiques et ce, surtout au cours des années 1990. Cette mouvance de l'argumentaire des partisans nous laisse songeur quant à l'objectif réel de la revendication. L'évolution du thème principal montre une certaine adaptation du projet face à un contexte changeant. Ainsi, l'amélioration constante ${ }^{11}$ des conditions routières présentes sur l'axe explique en partie la diminution du nombre d'accidents survenu sur la route 175. Ce résultat représentait l’objectif avoué par l'action collective : celui d'améliorer la sécurité dans la réserve faunique des Laurentides. Face à un projet de plus en plus difficile à justifier uniquement avec la notion de sécurité, la diversification de l'argumentaire s’avérait nécessaire pour la survie du projet. L'aspect intangible que sous-tendent les retombées socio-économiques des infrastructures de transport permettait cette survivance. Les impacts possibles de la réfection de cet axe routier autorisaient le prolongement du débat.

Cependant, les enjeux soutenus par le dossier, bien que soulevant les sensibilités de l'ensemble de la région, n’ont pu être universalisés au sein de la population du Saguenay-Lac-Saint-Jean. Bien que la cause ait rejoint l'ensemble de la région, l'incapacité de mobiliser les forces en présence a joué en défaveur du projet. Ce manque important a provoqué une détérioration des relations entre l'action collective et les organismes en charge du développement du réseau routier régional. Ce faisant, le projet a vu son influence se localiser davantage dans la sous-région du Saguenay.

En plus de ne pas faire l'unanimité au sein même de la région, le projet ne pouvait faire l'objet d'appuis importants à l'extérieur du Saguenay-Lac-Saint-Jean. Compte tenu des bénéfices considérés comme presque exclusifs à la région, la revendication ne put trouver écho ailleurs au Québec. Même la région de Québec, qui avait appuyé le projet à la fin des années 1980, s'est faite plus discrète après la réfection de la route 73 dans le secteur de Stoneham.

L'acceptation du projet est en grande partie attribuable à l'action des politiciens en place au Saguenay. Par leurs actions auprès de leur gouvernement respectif, ils ont effectué une grande conscientisation en regard au projet de doublement de la route 175 . Néanmoins, un certain opportunisme politique est palpable. L'enjeu que représentait la région pour les Libéraux fédéraux et provinciaux n'est pas étranger aux débouchés qu’a connus le projet depuis 1998.

\section{En guise de conclusion}

Actuellement, l'Union québécoise pour la conservation de la nature (UQCN) critique vertement le projet de doublement de la route 175 . S'appuyant sur un argumentaire structuré autour des principes du développement durable, cet organisme remet en question la pertinence du projet, prétextant le coût de l'infrastructure en regard de la capacité de payer des Québécois. Ainsi, selon l'UQCN, « l'investissement en cause [évalué entre 1,3 et 1,6 milliard de dollars par le regroupement] ne répond pas à des besoins économiques, sociaux et de sécurité ${ }^{12}$. Cette réaction de l'UQCN fait suite à l'engagement ferme du ministre du Développement durable, de l'Environnement et des Parcs de réaliser le projet de doublement de la route 175, un engagement survenu à l'aube des audiences du BAPE.

Cet épisode constitue un précédent dans la longue histoire de la revendication car, pour la première fois, une voix discordante s'élève en opposition à l'opinion populaire saguenayenne. Cette contestation actuellement menée par l'UQCN, par le débat qu'elle crée (ou plutôt qu'elle tente de créer), soulève des questions fondamentales sur l'action collective et l'objet de revendication. Comme nous avons pu le voir, l'annonce 
du projet de doublement de la route 175 fait suite à une longue histoire teintée de déterminisme. L'amélioration de la sécurité, véritable amorce du projet, s'est vue confinée uniquement dans la réalisation de la route à quatre voies divisées, comme s'il s'agissait de la seule solution possible et envisageable par l'action collective. Les considérations économiques ne sont venues qu'ajouter à un projet s'imposant par luimême.

\section{Les efforts investis dans la revendication de la route 175 ont-ils été dirigés vers la bonne cause, vers la bonne solution?}

Cette démarche menée par l'action collective se veut très sectorielle, ciblant un seul objet : une route à quatre voies divisées. Toutefois, nous sommes en droit de nous questionner sur la pertinence de ce projet face à la problématique régionale. Dans ce sens, les efforts investis dans la revendication de la route 175 , bien que démontrant une conscientisation de la population face aux problèmes (ou plutôt à une partie des problèmes) actuellement vécus, ont-ils été dirigés vers la bonne cause, vers la bonne solution ? La diversification de l'économie, la disparité des salaires et le faible entrepreneurship qui en découle ainsi que la faible présence du secteur secondaire dans le paysage économique régional, voilà quelques-uns des défis qui attendent la population du Saguenay-Lac-Saint-Jean au cours des années à venir. De plus, la réalisation de la route à quatre voies divisées risque maintenant de faire tomber dans l'oubli d'autres projets d'importance dans la région, comme par exemple la construction des derniers tronçons de la route 170 devant relier les villes d'Alma et La Baie ainsi que l'aménagement du réservoir sur la rivière Pikauba rendu nécessaire par les inondations de 1996. Dans cette perspective, il est aisé de comprendre que la gestion des deniers publics ne réponde pas uniquement à une logique de besoins, mais qu'elle est plutôt assujettie à une logique de priorité dans les besoins (dans laquelle s’ajoute souvent la subjectivité politique). La région semble déjà avoir fait son choix, mais est-ce le bon ?

\section{Notes et références}

1 L'auteur est diplômé à la maîtrise en urbanisme de l'Université de Montréal et est postulant au programme de troisième cycle en développement régional.
2 Syndrome «Pas dans ma cour» (not in my back yard): résistance sociale à accepter sur son territoire tout projet qui menacerait ou qui serait perçu comme une menace à la qualité de vie.

3 Nous n'avons qu'à penser à la mobilisation entourant le projet de la centrale du Suroît dans la ville de Beauharnois (centrale thermique fonctionnant au gaz naturel) qui a étendu le débat à l'ensemble de la province, ou encore le cas de l'autoroute 30 sur la Rive Sud de Montréal.

4 Proulx, M-U. (2004). L'impasse actuelle de l'économie urbaine et régionale du Saguenay-Lac-Saint-Jean : éléments de prospective, Saguenay, document présenté lors du colloque de Vision Saguenay 2025 «Le devenir de Saguenay ».

5 Le groupe Accès-Bleuets organisa une manifestation dans la réserve faunique des Laurentides. Ainsi, un cortège d'une centaine d'automobiles roulant à $50 \mathrm{~km} / \mathrm{h}$ arpenta la route 175 , créant un immense bouchon de circulation.

6 Groupe LCL (1991). Étude d'opportunité d'amélioration $d u$ lien routier entre la région du Saguenay-Lac-SaintJean et la région de Québec, Québec, Ministère des Transports.

7 Coentreprise BUC (1998). Construction d'une route à chaussées séparées dans la réserve faunique des Laurentides, Québec, Ministère des Transports.

8 Il est important de préciser que, lors de l'annonce officielle, un accord verbal avait été négocié entre les gouvernements provincial et fédéral. La concrétisation de l'entente entre les deux gouvernements fut le prétexte pour la seconde annonce.

$9 \quad$ Les projets identifiés au sein du Conseil régional de concertation et de développement (CRCD) étaient le parachèvement de la route 170 entre les villes d'Alma et La Baie, la réfection de la route 169 ceinturant le Lac-Saint-Jean, l'amélioration du tronçon de la route 169 situé dans la réserve faunique des Laurentides et de la route 155, l'aménagement de la route menant à la Baie James et ensuite le projet de la route 175 .

10 BAPE (1988). Le prolongement de l'autoroute 73 vers Stoneham, Québec.

11 Bien qu'il s'agisse d'un élément important, d'autres facteurs pourraient être considérés pour expliquer l'amélioration du bilan routier sur l'axe 175 . Nous pouvons mentionner la meilleure qualité des mécanismes de sécurité présents sur les véhicules (coussins gonflables, par exemple), l'augmentation de la surveillance policière et une plus grande conscientisation des automobilistes face aux dangers reliés à la vitesse. Toutefois, il s’agit là d’hypothèses.

12 Union québécoise pour la conservation de la nature (2005), Mémoire présenté dans le cadre des consultations sur les projets d'amélioration de la route 175 des kilomètres 60 à 84 et 84 à 227, mai. 